\title{
Characterizing Perceptual Artifacts in Compressed Video Streams
}

\author{
Kai Zeng, Tiesong Zhao, Abdul Rehman and Zhou Wang \\ Dept. of Electrical \& Computer Engineering, University of Waterloo, Waterloo, ON, Canada
}

\begin{abstract}
To achieve optimal video quality under bandwidth and power constraints, modern video coding techniques employ lossy coding schemes, which often create compression artifacts that may lead to degradation of perceptual video quality. Understanding and quantifying such perceptual artifacts play important roles in the development of effective video compression, streaming and quality enhancement systems. Moreover, the characteristics of compression artifacts evolve over time due to the continuous adoption of novel coding structures and strategies during the development of new video compression standards. In this paper, we reexamine the perceptual artifacts created by standard video compression, summarizing commonly observed spatial and temporal perceptual distortions in compressed video, with emphasis on the perceptual temporal artifacts that have not been well identified or accounted for in previous studies. Furthermore, a floating effect detection method is proposed that not only detects the existence of floating, but also segments the spatial regions where floating occurs*.
\end{abstract}

Keywords: video compression, video quality assessment, compression artifact, H.264-MPEG4/AVC, HEVC, flickering, floating detection

\section{INTRODUCTION}

The demand for high-performance network video communications has been increasing exponentially in recent years. According to Cisco Visual Networking Index, the sum of all forms of video (TV, VoD, Internet, and P2P) will constitute approximately 90 percent of global consumer traffic by $2015 .{ }^{1}$ A high-performance video compression technology is critical for current industrial video communication systems to catch up with such increasing demand. A fundamental issue in the design of video compression systems is to achieve an optimal compromise between the availability of resources (i.e. bandwidth, power, and time) and the perceptual quality of the compressed video. The constraint in available resources often leads to degradations of perceptual quality by introducing compression artifacts in the decoded video. For example, large quantization step could reduce power consumption, encoding time, as well as the bandwidth needed to encode the video, but, unfortunately, also results in video quality degradation.

Consumers' expectations for better Quality-of-Experience (QoE) nowdays has been higher than ever before. Despite the fast technological development in telecommunication and display devices, poor video quality originated from compression and streaming processes has disappointed a large volume of consumers, resulting in major revenue lost in digital media communication industry. Based on a recent viewer experience study, ${ }^{2}$ "In 2012, global premium content brands lost $\$ 2.16$ billion of revenue due to poor quality video streams and are expected to miss out on an astounding $\$ 20$ billion through $2017 "$. The poor video quality keeps challenging the viewers' patience and becomes a core threat to the video service ecosystem. According to the same study, ${ }^{2}$ roughly $60 \%$ of all video streams experienced quality degradation in 2012 . In another recent study, ${ }^{3} 90.4 \%$ interviewers reported "end-user video quality monitoring as either "critical", "very important", or "important" to their video

Further author information: (Send correspondence to Kai Zeng)

Kai Zeng: E-mail: kzeng@uwaterloo.ca, Telephone: 15198884567 ext. 31449

Tiesong Zhao: E-mail: ztiesong@uwaterloo.ca, Telephone: 15198884567 ext. 31448

Abdul Rehman: E-mail: abdul.rehman@uwaterloo.ca, Telephone: 15198884567 ext. 31449

Zhou Wang: E-mail: zhou.wang@uwaterloo.ca, Telephone: 15198884567 ext. 35301

*Image and video examples that demonstrate various types of spatial and temporal compression artifacts are available at https://ece.uwaterloo.ca/ z70wang/research/compression_artifacts/. 
initiatives", and almost half of the customer phone calls is related to video quality problems in Video-on-Demand (VOD) services and HDTV. Additionally, even though 58.1\% of the interviewed subjects reported the end-user QoE is "critical" and requires to be monitored, only $31 \%$ said they use network monitoring tools to discover quality problems. ${ }^{3}$ Therefore, there is an urgent need of effective and efficient objective video quality assessment (VQA) tools in current media network communication systems that can provide reliable quality measurement of end users' visual QoE.

Since compression is a major source of video quality degradation, we focuses on perceptual artifacts generated by standard video compression techniques in the current work. Various types of artifacts created by standard compression schemes had been summarized previously. ${ }^{4}$ Objective VQA techniques had also been designed to automatically evaluate the perceptual quality of compressed video streams. ${ }^{5}$ However, recent studies suggest that widely recognized VQA models (though promising) only achieve limited success in predicting the perceptual coding gain between state-of-the-art video coding techniques, and problems often occur when specific temporal artifacts appear in the compressed video streams. ${ }^{6}$ This is likely due to the adoption of the novel coding structures and strategies in the latest development of video compression standards such as H.264/AVC ${ }^{7}$ and the high efficiency video coding (HEVC). ${ }^{8}$ This motivates us to reexamine the perceptual artifacts created by video compression, with emphasis on the perceptual temporal artifacts that have not been well identified or accounted for in previous studies.

In this paper, we first attempt to elaborate various spatial and temporal artifacts originated from standard video compression. These include both conventional artifacts and those emerged recently in the new coding standards, such as various flickering and floating effects. Examples are provided to demonstrate the artifacts in different categories. Possible reasons and consequences of these artifacts together with their perceptual impact are discussed in the context of compression. Finally, an objective floating artifact detection scheme is proposed, which not only detects the existence of floating, but also indicates the location of floating regions in each video frame.

\section{PERCEPTUAL ARTIFACTS IN COMPRESSED VIDEO}

A diagram that summarizes various types of compression artifacts is given in Fig. 1. Both spatial and temporal artifacts may exist in compressed video, where spatial artifacts refer to the distortions that can be observed in individual frames while temporal artifacts can only be seen during video playback. Both spatial and temporal artifacts can be further divided into categories and subcategories of more specific distortion types. A detailed description of the appearance and causes of each type of perceptual compression artifacts will be given in the following sections. In addition to these artifacts, there are a number of other perceptual video artifacts that are often seen in real-world visual communication applications. These include those artifacts generated during video acquisition (e.g., camera noise, camera motion blur, and line/frame jittering), during video transmission in error-prone networks (e.g., video freezing, jittering, and erroneously decoded blocks caused by packet loss and delay), and during video post-processing and display (e.g., post deblocking and noise filtering, spatial scaling, retargeting, chromatic aberration, and pincushion distortion). Since compression is not the main cause of these artifacts, they are beyond the major focus of the current paper.

\subsection{Spatial Artifacts}

Block-based video coding schemes create various spatial artifacts due to block partitioning and quantization. These artifacts include blurring, blocking, ringing, basis pattern effect, and color bleeding. They are detected without referencing to temporally neighboring frames, and thus can be better identified when the video is paused. Due to the complexity of modern compression techniques, these artifacts are interrelated with each other, and the classification here is mainly based on their visual appearance.

\subsubsection{Blurring}

All modern video compression methods involve a frequency transform step followed by a quantization process that often removes small amplitude transform coefficients. Since the energy of natural visual signals concentrate at low frequencies, quantization reduces high frequency energy in such signals, resulting in significant blurring effect in the reconstructed signals. Perceptually, blurring typically manifests itself as a loss of spatial details or 


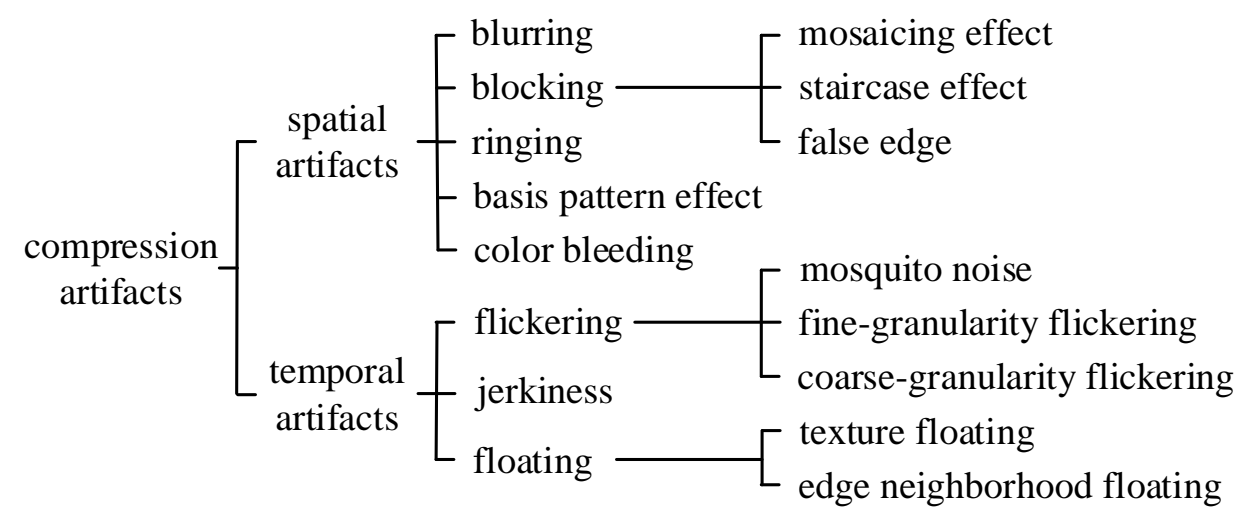

Figure 1. Categorization of perceptual artifacts created by video compression

sharpness at edges or texture regions in the image. Since in block-based coding schemes, frequency transformation and quantization are usually conducted within individual image blocks, blurring caused by such processes is often created inside the blocks.

Another source of blurring effect is in-loop de-blocking filtering, which is employed to reduce the blocking artifact across block boundaries, and are adopted as options by state-of-the-art video coding standards such as H.264/AVC and HEVC. The de-blocking operators are essentially spatially adaptive low-pass filters that smooth the block boundaries, and thus produces perceptual blurring effect.

A visual example is given in Fig. 2, where the left picture is a reference frame extracted from the original video, and the middle and right pictures are two decoded H.264/AVC frames with the de-blocking filter turned off and on, respectively. It can be observed that without de-blocking filtering, the majority of blur occurs within each block while the blocking artifact across the block boundaries is quite severe, for example, in the marked rectangular region in Fig. 2(b). When the de-blocking filter is turned on, much smoother luminance transition is observed in the same region, as shown in Fig. 2(c), but the overall appearance of the picture is more blurry.

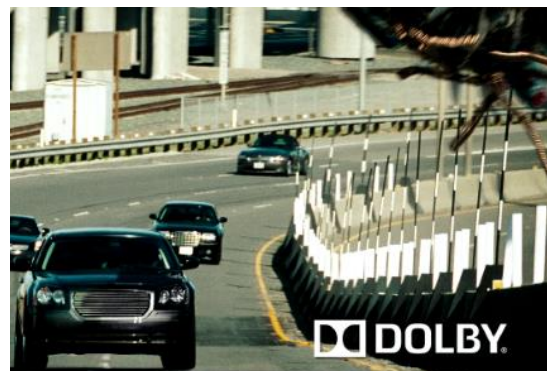

(a)

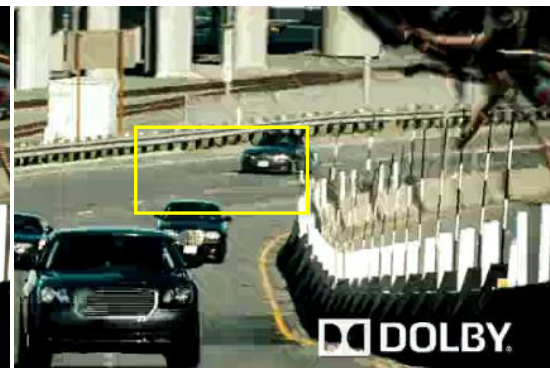

(b)

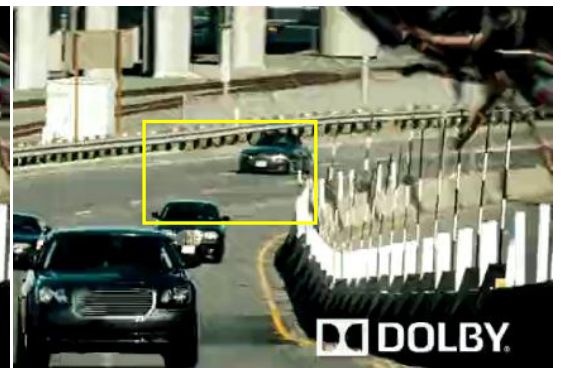

(c)

Figure 2. An example of spatial artifacts created by video compression. (a) Reference frame; (b) Compressed frame with de-blocking filter turned off; (c) Compressed frame with de-blocking filter turned on.

\subsubsection{Blocking}

Blocking artifact or blockiness is a very common type of distortion frequently seen in reconstructed video produced by video compression standards, which use blocks of various sizes as the basic units for frequency transformation, quantization and motion estimation/compensation, thus producing false discontinuities across block boundaries. Although all blocking effects are generated because of similar reasons mentioned above, their visual appearance may be different, depending on the region where blockiness occurs. Therefore, here we further classify the blocking effects into three subcategories. 


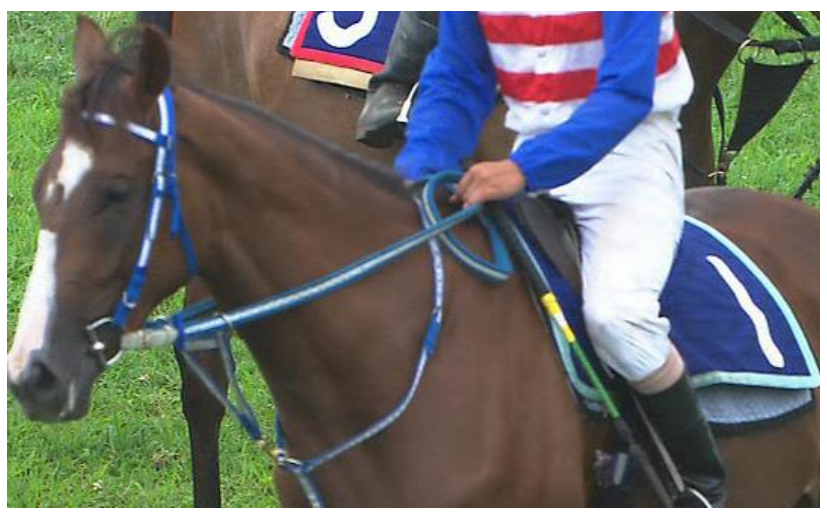

(a)

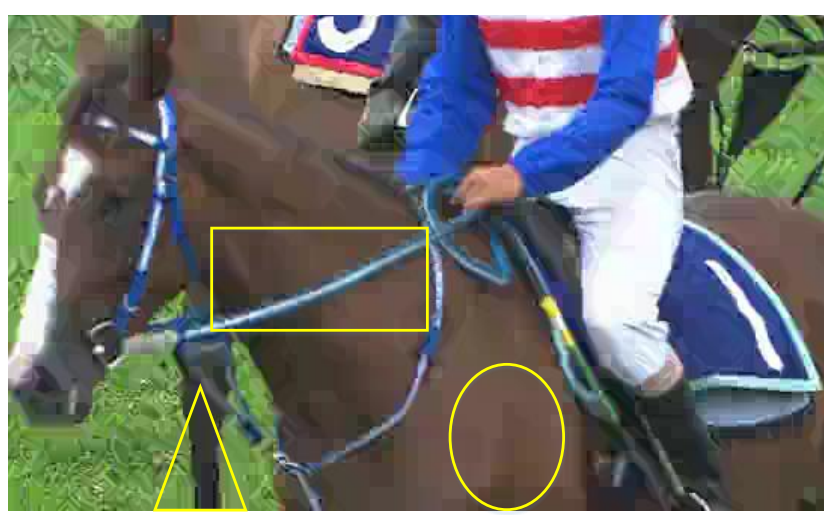

(b)

Figure 3. An example of blocking artifacts. (a) Reference frame; (b) Compressed frame with three types of blocking artifacts: mosaic effect (elliptical region); staircase effect (rectangular region); false edge (triangular region).

- Mosaic effect usually occurs when there is luminance transitions at large low-energy regions (e.g., walls, black/white boards, and desk surfaces). Due to quantization within each block, nearly all AC coefficients are quantized to zero, and thus each block is reconstructed as a constant DC block, where the DC values vary from block to block. When all blocks are put together, mosaic effect manifests as abrupt luminance change from one block to another across the space. The mosaic effect is highly visible and annoying to the visual system, where the visual masking effect (which stands for the reduced visibility of one image component due to the existence of another neighboring image component) is the weakest at smooth regions. An example is shown in the marked elliptical region in Fig. 3(b).

- Staircase effect typically happens along a diagonal line or curve, which, when mixed with the false horizontal and vertical edges at block boundaries, creates fake staircase structures. In Fig. 3(b), an example of staircase effect is highlighted in the marked rectangle region.

- False edge is a fake edge that appears near a true edge. This is often created by a combination of motion estimation/compensation based inter-frame prediction and blocking effect in the previous frame, where blockiness in the previous frame is transformed to the current frame via motion compensation as artificial edges. An example is given in the triangle marked region in Fig. 3(b).

\subsubsection{Ringing}

Sharp transitions in images such as strong edges and lines are transformed to many coefficients in frequency domain representations. The quantization process results in partial loss or distortion of these coefficients. When the remaining coefficients are combined to reconstruct the edges or lines, artificial wave-like or ripple structures are created in nearby regions, known as the ringing artifacts. Such ringing artifacts are most significant when the edges or lines are sharp and strong, and when the regions near the edges or lines are smooth, where the visual masking effect is the weakest. Fig. 4(b) shows an example of ringing artifacts. It is worth noting that when the ringing effect is combined with object motion in consecutive video frames, a special temporal artifact called mosquito noise is observed, which will be discussed later.

\subsubsection{Basis pattern effect}

The origin of the basis pattern effect is similar to that of the ringing effect, but the spatial regions where the basis pattern effect occurs are not restricted to sharp edges or lines. More specifically, in certain texture regions with moderate energy, when the transform coefficients are quantized, there is a possibility that only one transform coefficient remains (while all other coefficients are quantized to zero or nearly zero). As a result, when the image signal is reconstructed using a single coefficient, the basis pattern (e.g., a DCT basis) associated with the coefficient is created as a representation of the image structure. An example is shown in Fig. 5(b), in which the 


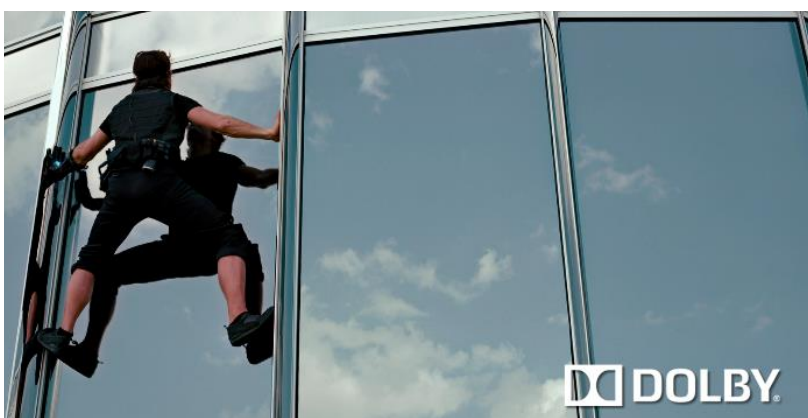

(a)

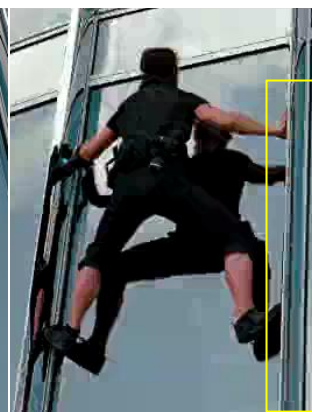

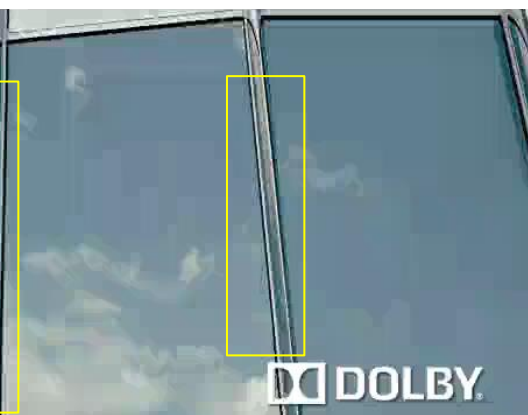

(b)

Figure 4. An example of ringing artifact. (a) Reference frame; (b) Compressed frame with ringing artifact.

basis pattern effect is highlighted in the marked rectangular regions. Since the basis pattern effect usually occurs at texture regions, its visibility depends on the nature of the texture region. If the region is in the foreground and attract visual attention, the basis pattern effect will have strong impact on perceived video quality. By contrast, if the region is in the background and does not attract visual attention, then the effect is often ignored by human observers.

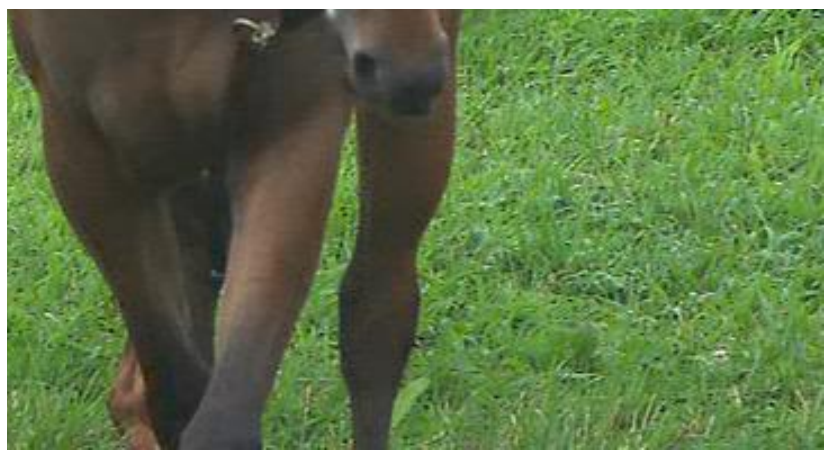

(a)

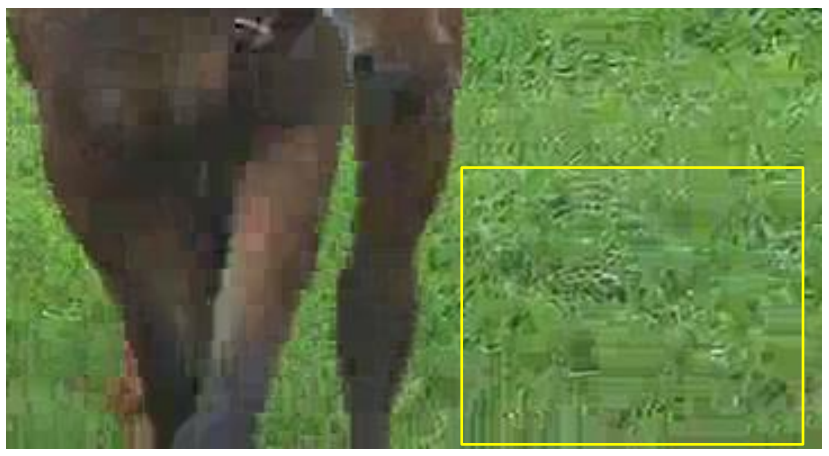

(b)

Figure 5. An example of basis pattern effect. (a) Reference frame; (b) Compressed frame with basis pattern effect.

\subsubsection{Color bleeding}

Color bleeding is a result of inconsistent image rendering across the luminance and chromatic channels. For example, in the most popular $\mathrm{YCbCr}$ 4:2:0 video format, the color channels $\mathrm{Cb}$ and $\mathrm{Cr}$ have half resolution of the luminance channel $\mathrm{Y}$ in both horizontal and vertical dimensions. After compression, all luminance and chromatic channels exhibit various types of distortions (such as blurring, blocking and ringing described earlier), and more importantly, these distortions are inconsistent across color channels. Moreover, because of the lower resolution in the chromatic channels, the rendering processes inevitably involve interpolation operations, leading to additional inconsistent color spreading in the rendering result. In the literature, it was shown that chromatic distortion is helpful in color image quality assessment, ${ }^{9}$ but how color bleeding affects the overall perceptual quality of compressed video is still an unsolved problem. An example of color bleeding is given in the highlighted elliptical region in Fig. 6(b).

\subsection{Temporal Artifacts}

Temporal artifacts refer to those distortion effects that are not observed when the video is paused but during video playback. Temporal artifacts are of particular interest to us for two reasons. First, as compared to spatial artifacts, temporal artifacts evolve more significantly with the development of video coding techniques. 


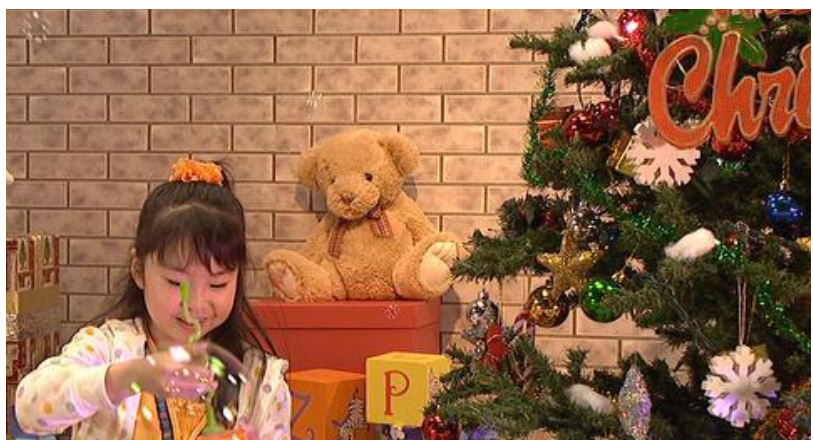

(a)

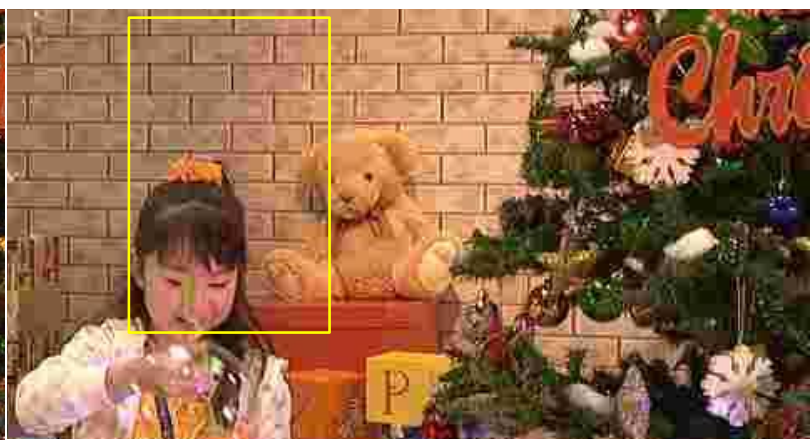

(b)

Figure 6. An example of color bleeding artifact. (a) Reference frame; (b) Compressed frame with color bleeding.

For example, texture floating did not appear to be a significant issue in early video coding standards, and is more manifest in H.264/AVC video, but is largely reduced in the latest HEVC coded video. Second, objective evaluation of such artifacts is more challenging, and popular VQA models often fail to account for these artifacts. For example, in our previous work, ${ }^{6}$ we demonstrated that well-known VQA models such as SSIM, ${ }^{10}$ MS-SSIM, ${ }^{11}$ VQM, ${ }^{12}$ and MOVIE $^{13}$ provide reasonable prediction of the spatial quality of paused video frames, but their performance drops significantly in predicting the perceptual coding gain from H.264/AVC to HEVC. More importantly, it was pointed out that such drops are largely due to the lack of proper assessment in temporal artifacts such as flickering and floating (ghosting). ${ }^{6}$

\subsubsection{Flickering}

Flickering artifact generally refers to frequent luminance or chrominance changes along temporal dimension that does not appear in uncompressed reference video. In the literature, flickering has been identified as an important temporal artifacts that has significant impact on perceived video quality, and flickering reduction has been a major research topic in the video coding community. ${ }^{14,15}$ Nevertheless, the specific descriptions of flickering are sometimes inconsistent or even conflicting (e.g., some authors detect flickering in large background areas while others identify it near object boundaries). This might be due to the complexity and variations of flickering appearance. To clarify the definition of flickering, here we further divide it into three subcategories based on the frequency and spatial locations where flickering occurs, namely mosquito noise, fine-granularity flickering and coarse-granularity flickering.

- Mosquito noise is a joint effect of object motion and time-varying spatial artifacts (such as ringing and motion prediction error) near sharp object boundaries. Specifically, the ringing and motion prediction error are most manifest at the regions near the boundaries of objects. When the objects move, such noise-like time-varying artifacts move together with the objects, and thus look like "mosquitos" flying around the objects. Since moving objects attract visual attention and the plane region near object boundaries have weak visual masking effect on the noise, mosquito noise is usually easily detected and has strong negative impact on perceived video quality.

- Coarse-granularity flickering refers to low-frequency sudden luminance changes in large spatial regions that could extend to the entire video frame. The most likely reason of such flickering is the use of groupof-picture (GoP) structures in standard video compression techniques. For example, in an I-P-P-...-P GoP structure, the first frame, called I-frame is coded independently using intra-coding method, while all other frames, called P-frames, are coded after predictions from the previous frames. The length of a GoP is limited, and thus when a new GoP starts, there is no dependency between the last P-frame in the previous GoP and the I-frame in the current GoP, and thus sudden luminance change is likely to be observed, especially when these two frames are about the same scene. As a result, the frequency of coarse-granularity flickering is typically determined by the size of GoP. Advanced video encoders may not 
use fixed GoP lengths or structures, and an I-frame may be employed only when scene change occurs, and thus coarse-granularity flickering may be avoided or significantly reduced.

- Fine-granularity flickering is typically observed in large low- to mid-energy regions with significant blocking effect and slow motion. In these regions, significant blocking effect occurs at each frame, and because of the variations between frames (due to either motion or texture details), the levels of blockiness and the DC values in corresponding blocks change frame by frame. Consequently, these regions appear to be flashing at high frequencies (frame-by-frame as opposed to GoP-by-GoP in coarse-granularity flickering). Such flashing effect is highly eye-catching and perceptually annoying, especially when the associated moving regions are of interest to the human observers.

\subsubsection{Jerkiness}

Jerkiness occurs when the temporal resolution is not high enough to catch up with the speed of moving objects, and thus the object motion appears to be discontinuous. Traditionally, jerkiness is not considered a compression artifact, but an effect of the low temporal resolution of video acquisition device, or a video transmission issue when the available bandwidth is not enough to transmit all frames and some frames have to be dropped or delayed. In state-of-the-art video coding standards, especially when temporally scalable video coding and hierarchical frame prediction structures are employed, the video frames are naturally divided into layers that correspond to coarseto-fine temporal resolutions. When the fine-resolution layers are dropped (e.g., due to bandwidth constraint), potential jerkiness becomes a concern. The highly visible jerkiness is typically observed only when there is strong object motion in the frame. The resulting unsmooth object movement may cause significant perceptual quality degradation.

\subsubsection{Floating}

Floating refers to the appearance of illusive motion in certain regions as opposed to their surrounding background. The illusive motion is erroneous because these regions are supposed to stay or move together with the background. Typically, the video encoders associate the Skip coding mode with these regions (where the encoding of motioncompensated prediction residue is skipped), and thus the structural details within the regions keep unchanged across frames, which is again erroneous as the actual details in the regions are evolving over time. Visually these regions create a strong perceptual illusion as if they were floating on top of the surrounding background. Depending on the type of region where floating occurs, it can be further classified into texture- and edge-floating.

- Texture floating typically occurs at large mid-energy texture regions. The most common case is when a scene with large textured regions such as water surface or trees is captured with slow camera motion. Despite the actual shifting of image content due to camera motion, many video encoders choose to encode the blocks in the texture regions with zero motion and Skip mode. These are reasonable choices to save bandwidth without significantly increasing the mean squared error or mean absolute error between the reference and reconstructed frames, but often create strong texture floating illusion. Not surprisingly, such floating illusive motion is usually in opposite direction with respect to the camera motion with the same absolute speed. In previous work, it was sometimes also referred to as "ghosting". ${ }^{6}$

- Edge neighborhood floating is observed in stationary regions that are next to the boundaries of moving objects. Rather than remaining stationary, these regions may move together with the boundaries of the objects. As in texture floating, the major source of this effect is likely the use of Skip mode, which simply copies the regions surrounding object boundaries from one frame to another without any further update in image details. Different from texture floating, edge neighborhood floating may appear without global motion. It is often visually unpleasant because it looks like there exists a wrapped package surrounding and moving together with the object boundaries. Previously, this effect was also called stationary area temporal fluctuations. ${ }^{4}$ 


\section{TEXTURE FLOATING DETECTION}

Among all types of temporal artifacts, texture floating is perhaps the least identified in the literature, but in our study, is found to be highly eye-catching and visually annoying when it exists. Moreover, to the best of our knowledge, no existing objective VQA method provides reliable assessment of texture floating.

According to our empirical observations, the following factors are relevant in detecting and assessing texture floating.

- Global motion: Texture floating is typically observed in the video frames with global camera motion, including translation, rotation and zooming. The relative motion between the floating regions and the background creates the floating illusion in the visual system. In our algorithm, a robust global motion estimation method is employed which uses the statistical distribution of motion vectors from the compressed bitstream. $^{16}$

- Skip mode: As described in Section 2.2.3, the use of Skip mode is the major source of temporal floating effect. When the compressed video stream is available, the Skip mode can be easily detected in the syntax information.

- Local energy: In high-energy texture and edge regions, erroneous motion estimation and Skip mode selection are unlikely in most high-performance video encoders. On the other hand, there is no visible texture in low-energy regions. Therefore, texture floating is most likely seen in mid-energy regions. Therefore, we define two threshold energy parameters $E_{1}, E_{2}$ in our algorithm to constrain the energy range for texture floating detection.

- Local luminance: The visibility of texture floating is also limited by the luminance levels of the floating regions. Because human eyes are less sensitive to textures in highly bright or dark regions, we define two threshold luminance parameters $L_{1}, L_{2}$ to constrain our algorithm to consider only mid-luminance regions for texture floating identification.

- Temporal variation similarity: As discussed in Section 2.2.3, temporal floating is often associated with erroneous motion estimation. In the reconstruction of video frames, erroneous motion estimation/compensation leads to significant distortions along temporal direction. In the case that the original uncompressed video is available to compare with, it is useful to evaluate the similarity of temporal variation between the reference video frames and the compressed video frames as a factor to detect temporal floating effect. Let $\left\{x_{1}, x_{2}\right\}$ and $\left\{y_{1}, y_{2}\right\}$ be two consecutive video frames extracted from the reference and compressed video, respectively. We evaluate temporal variation similarity as $\operatorname{SSIM}\left(\left|x_{2}-x_{1}\right|,\left|y_{2}-y_{1}\right|\right)$, where $\operatorname{SSIM}($.$) is the SSIM measure. { }^{10}$ We can then define a upper threshold $Q_{1}$ to limit the search range in our texture floating detection algorithm.

Each of the above features constrains the potential regions in a video frame where texture floating may occur. This leads to a texture floating detection algorithm that identifies all the spatial regions that satisfy these constraints as texture floating regions.

Fig. 7 demonstrates the performance of the proposed algorithm. Fig. 7(a) is the original frame extracted from the "PO_Org.yuv" sequence from LIVE Mobile Video Quality Database. ${ }^{17}$ Fig. 7(b) is the compressed frame created by H.264 reference model JM18.3 with $Q P=42$, where the visual texture floating regions are marked manually (Note that precise marking of texture floating in a video frame is a tedious job for human subjects, and the result shown here only provides a rough reference on the regions detected as having perceptual texture floating). Fig. 7(c) is the texture floating map generated by the proposed method, where black regions indicate the existence of texture floating. It can be observed that the proposed algorithm not only detects the existence of texture floating, but also successfully locates such regions in the compressed video frame. 


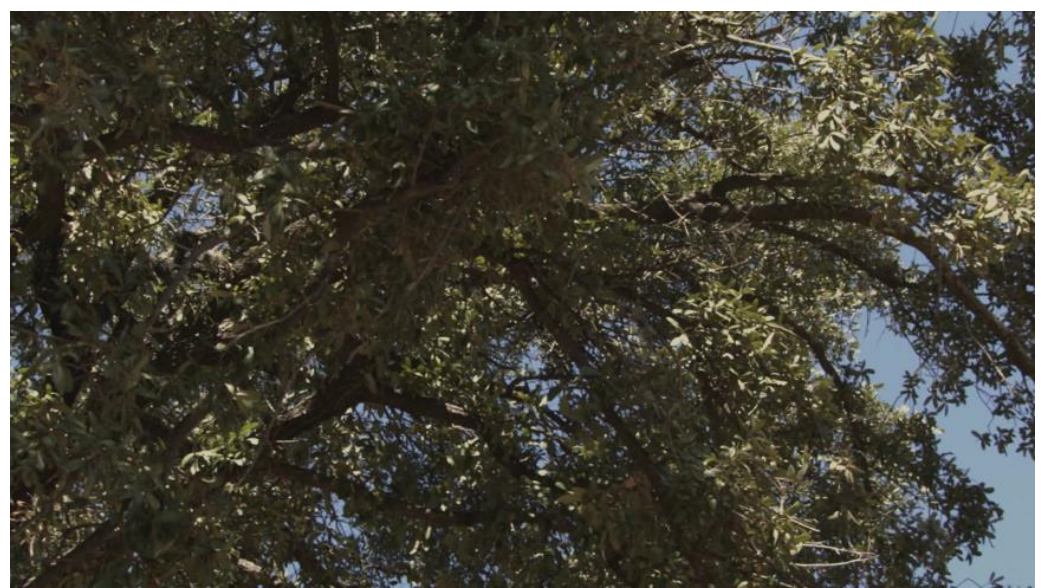

(a)

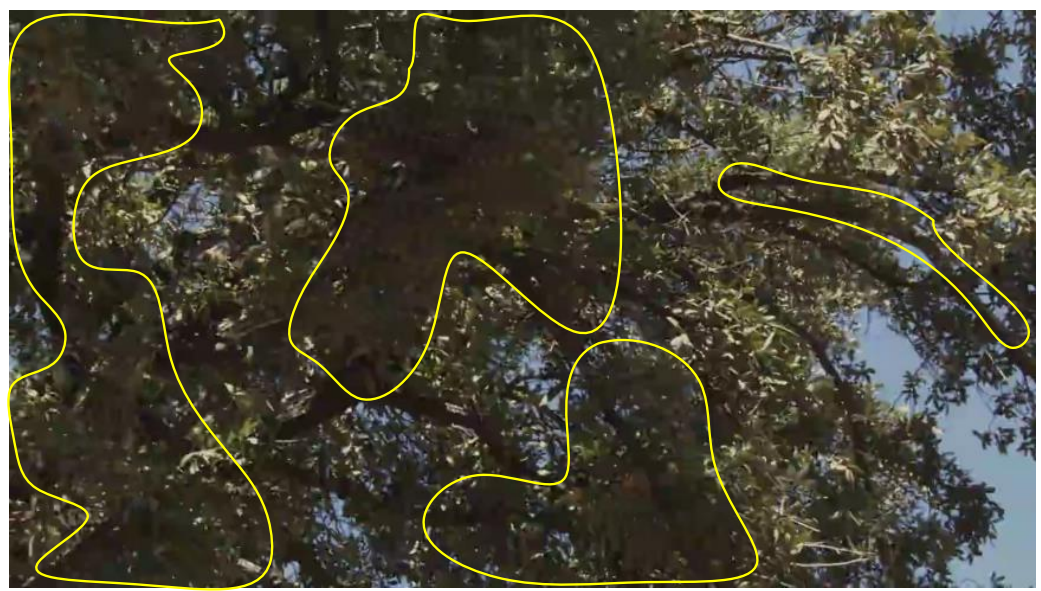

(b)

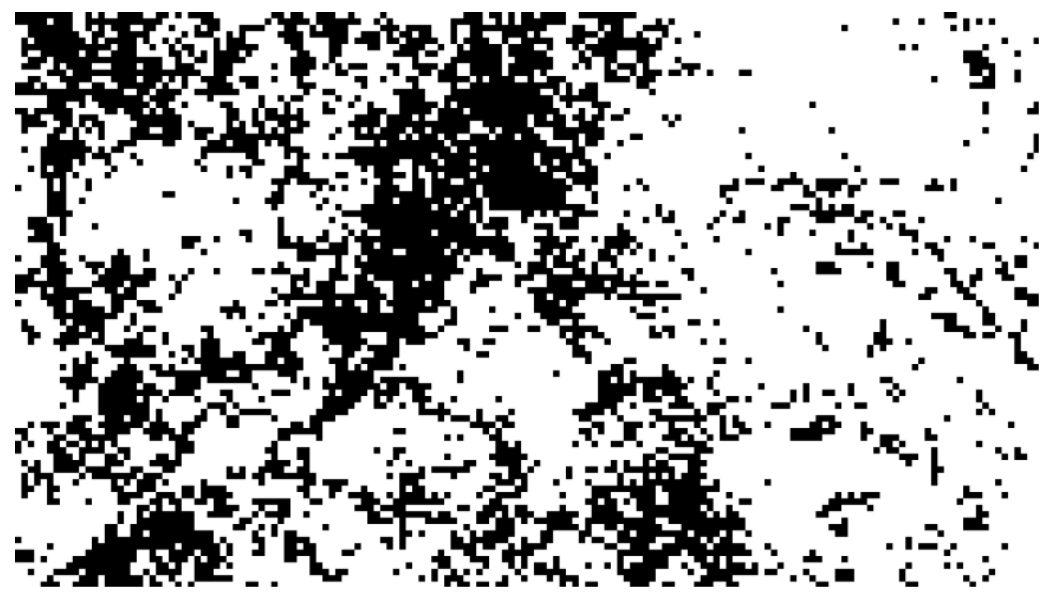

(c)

Figure 7. An example of texture floating detection. (a) The $200 t h$ frame in the original video; (b) The 200th frame in the compressed video (visual texture floating regions are marked manually); (c) The texture floating map generated by the proposed method (black regions indicate texture floating is detected). 


\section{CONCLUSION}

In this work, we reexamine perceptual artifacts created by state-of-the-art video compression technologies. We find it important to reclassify the artifacts observed in previous studies and also to identify new artifacts that are evolving with the development of novel video coding schemes. In particular, the fine classification, the new naming approach, and the corresponding descriptions of the temporal flickering and floating effects are new to the literature. Furthermore, we focus on the texture floating effect, which has been the least identified and investigated type of temporal artifact in the literature. Related features are identified and a novel objective floating artifact detection algorithm is proposed, which not only detects the existence of texture floating, but also locates the texture floating regions in each video frame.

The current work also lays out a work plan for future studies. Firstly, objective VQA methods need to be reexamined and further developed to detect each compression artifacts reliably and efficiently. Secondly, video encoders may be designed to eliminate or minimize the impact of these perceptual artifacts. Finally, pre- and post-processing techniques may also be developed to suppress or remove these compression artifacts.

\section{REFERENCES}

[1] Cisco Visual Networking Index: Forecast and Methodology, 2010-2015.

[2] Conviva, Viewer Experience Report, 2013.

[3] Symmetricom, Cable Operator Video Quality Study, 2008.

[4] Yuen, M. and Wu, H., "A survey of hybrid MC/DPCM/DCT video coding distortions," Signal Processing 70(3), 247-278 (1998).

[5] H. R. Wu and K. R. Rao, Ed., [Digital Video Image Quality and Perceptual Coding], CRC Press, Taylor \& Francis Group, Boca Raton (2005).

[6] Zeng, K., Rehman, A., Wang, J., and Wang, Z., "From H.264 to HEVC: Coding gain predicted by objective video quality assessment models," in [International Workshop on Video Processing and Quality Metrics for Consumer Electronics], (Feb 2013).

[7] Wiegand, T., Sullivan, G., Bjontegaard, G., and Luthra, A., "Overview of the H.264/AVC video coding standard," IEEE Trans. Circuits and Systems for Video Technology 13(7), 560-576 (2003).

[8] Sullivan, G., Ohm, J., Woo-Jin, H., and Wiegand, T., "Overview of the High Efficiency Video Coding (HEVC) standard," IEEE Trans. Circuits and Systems for Video Technology 22(12), 1649-1668 (2012).

[9] Redi, J., Gastaldo, P., Heynderickx, I., and Zunino, R., "Color distribution information for the reducedreference assessment of perceived image quality," IEEE Trans. Circuits and Systems for Video Technology 20(12), 1757-1769 (2010).

[10] Wang, Z., Bovik, A., Sheikh, H., and Simoncelli, E., "Image quality assessment: From error visibility to structural similarity," IEEE Trans. Image Processing 13(4), 600-612 (2004).

[11] Wang, Z., Simoncelli, E., and Bovik, A., "Multiscale structural similarity for image quality assessment," Conference Record of the Thirty-Seventh Asilomar Conference on Signals, Systems and Computers 2, 13981402 (Nov. 2003).

[12] Pinson, M. H., "A new standardized method for objectively measuring video quality," IEEE Trans. Broadcasting 50, 312-322 (Sept. 2004).

[13] Seshadrinathan, K. and Bovik, A. C., "Motion tuned spatio-temporal quality assessment of natural videos," 19, 335-350 (Feb. 2010).

[14] Fan, X., Gao, W., Lu, Y., and Zhao, D., "Flicking reduction in all intra-frame coding," in [Proc. 5th Meeting Joint Video Team ISO/IEC JTC1/SC29/WG11, ITU-T SG16 Q.6, document JVT-E070.doc], (Oct. 2002).

[15] Yang, J. X. and Wu, H. R., "Robust filtering technique for reduction of temporal fluctuation in H.264 video sequences," IEEE Trans. Circuits and Systems for Video Technology 20(3), 458-462 (2010).

[16] Haller, M., Krutz, A., and Sikora, T., "Robust global motion estimation using motion vectors of variable size blocks and automatic motion model selection," in [IEEE 17th International Conference on Image Processing], (Sept. 2010).

[17] Moorthy, A., Choi, L., Bovik, A., and deVeciana, G., "Video quality assessment on mobile devices: Subjective, behavioral and objective studies," IEEE Journal of Selected Topics in Signal Processing 6(6), 652-671 (2012). 